University of Nebraska - Lincoln

DigitalCommons@University of Nebraska - Lincoln

2-9-1998

\title{
Changes in Metallicity and Electronic Structure Across the Surface Ferroelectric Transition of Ultrathin Crystalline Poly(vinylidene Fluoride-Trifluoroethylene) Copolymers
}

\author{
Jaewu Choi \\ University of Nebraska-Lincoln, jchoi@ece.eng.wayne.edu \\ Peter A. Dowben \\ University of Nebraska-Lincoln, pdowben@unl.edu \\ Shawn Pebley \\ University of Nebraska-Lincoln
}

\author{
A.V. Bune \\ University of Nebraska-Lincoln \\ Stephen Ducharme \\ University of Nebraska, sducharme1@unl.edu \\ See next page for additional authors \\ Follow this and additional works at: https://digitalcommons.unl.edu/physicsdowben \\ Part of the Physics Commons
}

Choi, Jaewu; Dowben, Peter A.; Pebley, Shawn; Bune, A.V.; Ducharme, Stephen; Fridkin, V.M.; Palto, S.P.; and Petukhova, N., "Changes in Metallicity and Electronic Structure Across the Surface Ferroelectric Transition of Ultrathin Crystalline Poly(vinylidene Fluoride-Trifluoroethylene) Copolymers" (1998). Peter Dowben Publications. 39.

https://digitalcommons.unl.edu/physicsdowben/39

This Article is brought to you for free and open access by the Research Papers in Physics and Astronomy at DigitalCommons@University of Nebraska - Lincoln. It has been accepted for inclusion in Peter Dowben Publications by an authorized administrator of DigitalCommons@University of Nebraska - Lincoln. 


\section{Authors}

Jaewu Choi, Peter A. Dowben, Shawn Pebley, A.V. Bune, Stephen Ducharme, V.M. Fridkin, S.P. Palto, and N. Petukhova 


\title{
Changes in Metallicity and Electronic Structure Across the Surface Ferroelectric Transition of Ultrathin Crystalline Poly(vinylidene Fluoride-Trifluoroethylene) Copolymers
}

\author{
Jaewu Choi, P. A. Dowben,* Shawn Pebley, A. V. Bune, and Stephen Ducharme \\ Department of Physics, Behlen Laboratory of Physics, Center for Materials Research and Analysis, \\ University of Nebraska-Lincoln, Lincoln, Nebraska 68588-0111 \\ V. M. Fridkin, S. P. Palto, and N. Petukhova \\ Institute of Crystallography, The Russian Academy of Sciences, 117333, Moscow, Russia
}

(Received 18 August 1997)

\begin{abstract}
The surface ferroelectric-paraelectric phase transition in crystalline copolymer films of vinylidene fluoride $(70 \%)$ with trifluoroethylene $(30 \%)$, at about $20{ }^{\circ} \mathrm{C}$, is accompanied by a dramatic change in both the electronic structure and metallicity. The temperature-dependent changes in the electronic structure occur primarily in the conduction band. This P(VDF-TrFE 70:30) material is observed to resemble an $n$-type semiconductor, and the change in electronic structure at the surface ferroelectric transition resembles a semiconductor-to-semimetal transition. [S0031-9007(98)05320-4]

PACS numbers: $77.80 . \mathrm{Bh}$
\end{abstract}

It has long been appealing to connect the ferroelectric transition to that of ferromagnetic ordering. At the most fundamental level, both transitions should have their origin in the electronic structure of the material. Recently, striking changes in electronic structure have been found to accompany the ferromagnetic transition in the doped manganese oxide perovskite materials $\mathrm{La}_{1-x} \mathrm{M}_{x} \mathrm{MnO}_{3}(M=$ $\mathrm{Ca}, \mathrm{Sr}, \mathrm{Ba})[1-3]$. While the inorganic ferroelectric crystalline oxides are in many respects similar to the manganese oxides, all complex oxides have surfaces that are difficult to prepare and characterize [4]. The crystalline ferroelectric polymers are molecular systems whose surface composition is well defined and provide, therefore, an ideal system in which to test if the changes in electronic structure accompany the ferroelectric transition.

Recently, a new first-order ferroelectric phase transition (as revealed by a pyroelectric anomoly, thermal hysteresis of the dielectric response and polarization hysteresis) was observed at $20^{\circ} \mathrm{C}$ in ferroelectric vinylidene-fluoride polymers, distinct from the bulk transition ferroelectric transition at $80{ }^{\circ} \mathrm{C}$ [5]. This new transition was ascribed to a surface ferroelectric transition, though the data could not be used to exclude the possibility that the transition was associated with the interior layers or buried interface. Enhanced surface ferromagnetism has been observed [6] and other related surface effects have been predicted [7], so a surface ferroelectric transition is not without precedent. Nonetheless, confirming that this new transition is indeed a surface transition is a goal of this paper.

The crystalline 5 monolayer (ML) films of poly (vinylidene fluoride-trifluoroethylene) [P(VDF-TrFE)], a copolymer of vinylidene fluoride with trifluoroethylene, consists of all trans- $\left(\left(-\mathrm{CH}_{2}-\mathrm{CF}_{2}\right)_{x}-\left(\mathrm{CF}_{2}-\mathrm{CHF}-\right)_{1-x}\right)_{n^{-}}$ chains as shown in Fig. 1(a). The films were formed by Langmuir-Blodgett monolayer deposition from water subphase as described elsewhere [8]. The films were deposited on silicon for photoemission studies and on an aluminum-coated glass substrate with a sputtered aluminum top electrode for dielectric studies. The inverse photoemission and photoemission studies were performed in a UHV chamber described in detail elsewhere [3] with a base pressure better than $1 \times 10^{-10}$ Torr. The films were prepared by gentle annealing to $200{ }^{\circ} \mathrm{C}$ in vacuo and surface composition characterized with core level spectroscopy. The valence band photoemission spectra were acquired with a helium lamp operated at the He I emission line $(21.2 \mathrm{eV})$ and a Physical Electronics model 365A hemispherical analyzer. The combined resolution, as determined from the Fermi edge of gold was $50 \mathrm{meV}$. For the inverse photoemission spectroscopy (IPES) studies, a Geiger-Müller detector with a $\mathrm{CaF}_{2}$ window was used in conjunction with an Erdman-Zipf electron gun [9]. The overall IPES energy resolution was $\sim 400 \mathrm{meV}$. All IPES

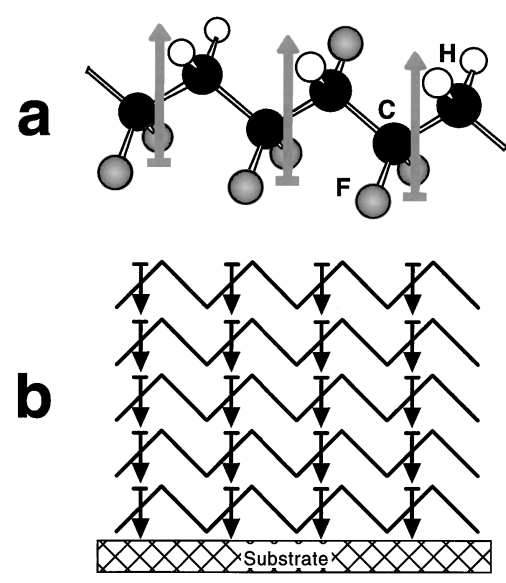

FIG. 1. A schematic diagram of the copolymer film of vinylidene fluoride $(70 \%)$ with trifluoroethylene $(30 \%)$. (a) The copolymer chain structure. (b) The stacking of a polarized 5 ML film. 
spectra were collected with the incident electrons normal to the surface. The Fermi level for both inverse photoemission and photoemission was established using a gold reference, and the temperature was determined using a chromel-alumel thermocouple. Dielectric measurements were undertaken at $1 \mathrm{kHz}$ with an $\mathrm{HP} 4192 \mathrm{~A}$ impedance analyzer. Conductance was measured at $0.1 \mathrm{~V}$ with a Keithly 498 current amplifier. The pyroelectric response was measured by recording the ac sample current due to heating from an Ar ion laser modulated at $1 \mathrm{kHz}$. The last three techniques are described in more detail elsewhere $[5,10]$.

In Fig. 2 we present a series of PES and IPES spectra of a 5 ML film of P(VDF-TrFE 70:30) (70\% vinylidene fluoride with $30 \%$ trifluoroethylene). As seen in Fig. 2, the inverse photoemission places the Fermi level near the unoccupied conduction band, while little or no density of states is observed near the Fermi energy in photoemission. Establishment of the Fermi level (the chemical potential) near the unoccupied density of states, the conduction band edge, is characteristic of an $n$-type semiconductor. With a film only $5 \mathrm{ML}$ thick, band bending is not likely to be a dominant effect, and we found no evidence for surface photovoltage effects.

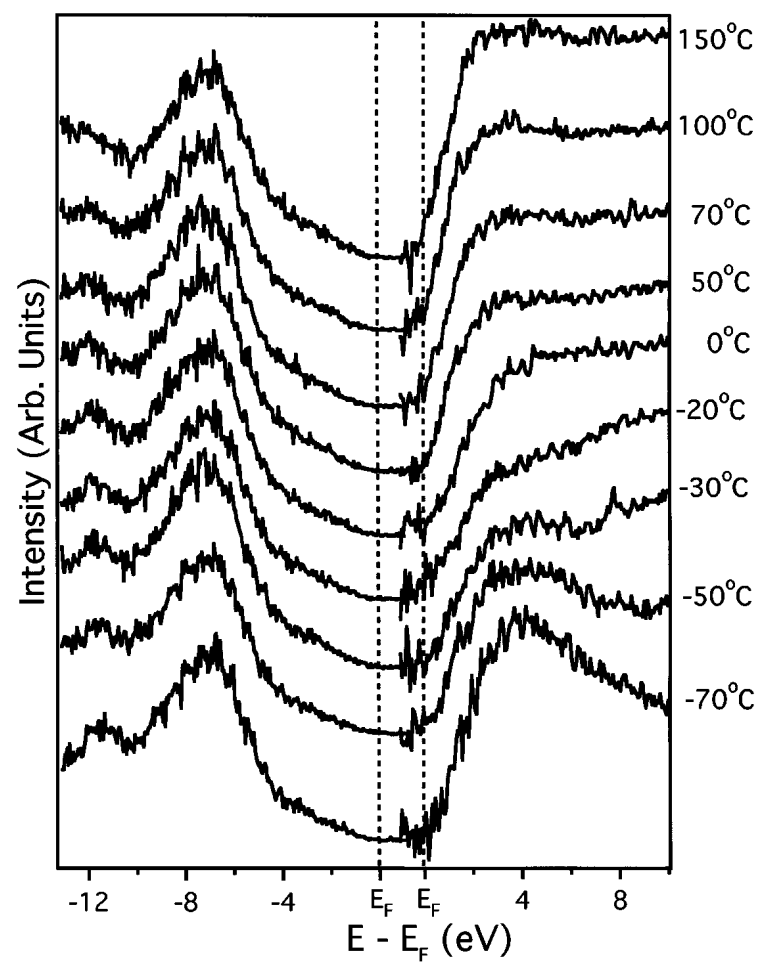

FIG. 2. Normal incidence inverse photoemission spectra (right) and normal emission photoemission spectra (left) of a 5 ML crystalline copolymer film of vinylidene fluoride $(70 \%)$ with trifluoroethylene (30\%). The spectra were taken as a function of temperature. Little difference was observed with the spectra taken with increasing temperature as opposed to decreasing temperature (no hysteresis).
The influence of temperature on the electronic structure in the $5 \mathrm{ML}$ ferroelectric copolymer film is quite pronounced in IPES, as seen in Fig. 2. With increased temperature, the unoccupied molecular orbitals of P(VDFTrFE) shift to lower binding energies, driving the conduction band into the Fermi level. Substantial changes in the unoccupied density of states are observed as a function of temperature in the vicinity of the Fermi energy, as summarized in Fig. 3(a). This result, taken in conjunction with the shift of the unoccupied density of states toward the Fermi level, as seen in Figs. 2 and 3(b), indicates that the insulator gap is closing with increasing temperature.

The increase in the observed density of states at the Fermi level [Fig. 3(a)] and the increasing binding energy of the lowest unoccupied molecular orbital (LUMO) states toward the Fermi level [Fig. 3(b)] in the crystalline ferroelectric polymer with increasing temperature coincide with an increase in the conductivity [Fig. 3(c)]. These changes all correspond to the ferroelectric transition observed at $20^{\circ} \mathrm{C}$ (on heating). No such similar dramatic change in the surface sensitive measurements [Figs. 3(a) and 3(b)] or the electronic structure is observed in the vicinity of the bulk ferroelectric transition at $\approx 80^{\circ} \mathrm{C}[5,8,10]$. The pyroelectric response [Fig. 3(d)] and capacitance [Fig. 3(e)] show anomalies at both the
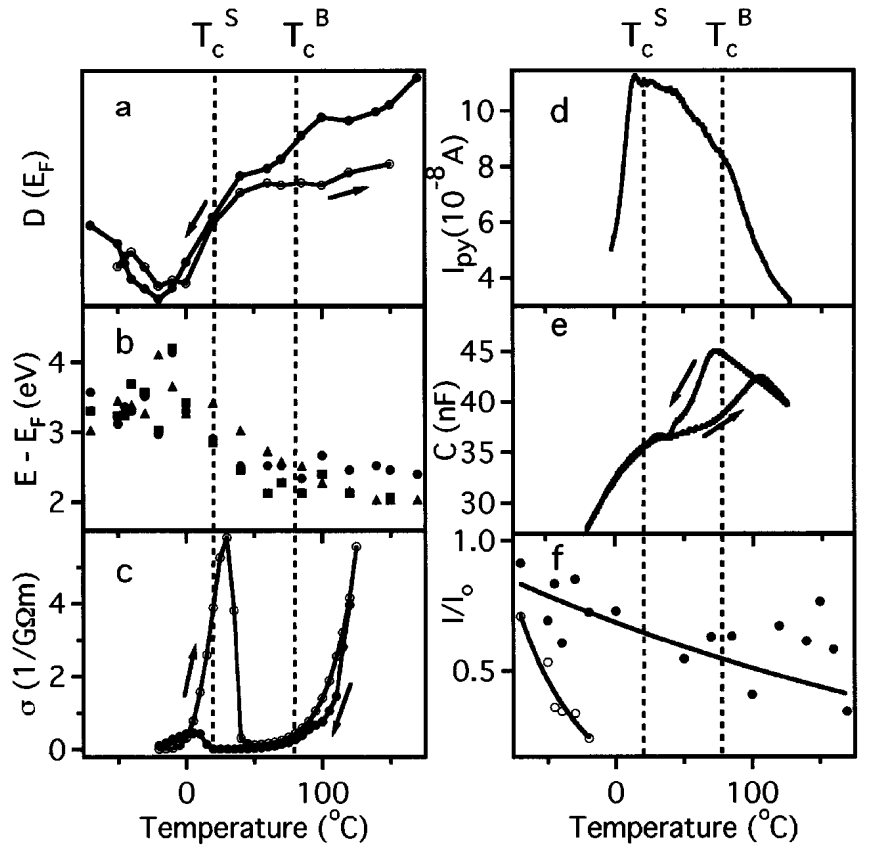

FIG. 3. The integrated density of states within a binding energy of $0.5 \mathrm{eV}$ above the Fermi level derived from inverse photoemission is shown in (a). The binding energy shift of the LUMO states above $E_{F}$ (b), the conductivity through a $5 \mathrm{ML}$ film (c), the pyroelectric current (d), the capacitance (e) are also shown for comparison. The intensity of the photoemission feature at $12 \mathrm{eV}$ binding energy $(O)$ and LUMO states $(\bigcirc)$ as a function of temperature are also shown, fitted to calculated intensity for an effective Debye temperature of 67 and $26 \mathrm{~K}$, respectively (f). 
$80{ }^{\circ} \mathrm{C}$ bulk phase transition temperature and at the $20^{\circ} \mathrm{C}$ surface phase transition temperature. The pyroelectric response identifies both transitions as involving ferroelectric phases, and the thermal hysteresis in the capacitance signifies a first-order phase transition. Additional evidence for the first-order surface ferroelectric phase transition at $20^{\circ} \mathrm{C}$ is presented elsewhere [5]. This type of change in the unoccupied density of states near the Fermi level has been observed before. For example, Na doping of films formed from molecular icosahedra [11] moves the conduction band edge also toward the Fermi energy with a concomitant increase in the density of states at the Fermi energy. This change is associated with a nonmetalto-metal transition with increasing alkali concentration [11]. The blue bronze materials show a similar movement of the valence band edge with temperature through the nonmetal-to-metal transition [12]. The changes in conductivity, as well as the increased density of states in the vicinity of the ferroelectric transition at about $20^{\circ} \mathrm{C}$, indicate that in the $\mathrm{P}(\mathrm{VDF}-\mathrm{TrFE})$ crystalline polymer film there is a similar change in metallicity. The surface ferroelectric phase $\left(T<20^{\circ} \mathrm{C}\right)$ is characteristically insulating with a clearly established band gap. Since we observe no increase in the occupied density of states (photoemission), we cannot conclusively say that the corresponding surface paraelectric phase $\left(T>20^{\circ} \mathrm{C}\right)$ is metallic but, given the changes in conductivity and electronic structure, it certainly resembles a semimetal. Thus this ferroelectric phase transition at about $20^{\circ} \mathrm{C}$ is accompanied by a change in electronic structure that closely resembles a nonmetal-to-metal transition in many respects.

Photoemission and inverse photoemission, like all electron spectroscopies, are very surface sensitive. The changes in electronic structure, primarily in the unoccupied density of states, are associated with the ferroelectric transitions at $\approx 20^{\circ} \mathrm{C}$ but not the accepted $[5,8,10]$ bulk transition at $\approx 80{ }^{\circ} \mathrm{C}$, as indicated in Fig. 3. This provides compelling additional evidence that the ferroelectric transition at $\approx 20{ }^{\circ} \mathrm{C}$ is associated with the surface layers [5], while the bulk ferroelectric transition is not strongly manifest at the surface. The increasing metallization of the surface across the approximately $20^{\circ} \mathrm{C}$ surface ferroelectric phase transition must lead to an increase in the dielectric response of the surface. Such an increase in the dielectric response in the surface region could suppress any manifestation of the nonmetallic ferroelectric phase in the bulk. This would make it very difficult to observe the bulk ferroelectric phase transition at $\approx 80{ }^{\circ} \mathrm{C}$, as is indeed the case (as indicated in Figs. 2 and 3).

Increasing metallization with increasing temperature has been observed for the surface layers of $\mathrm{Ge}(100)$ [13] and $\mathrm{Si}(100)$ [14] by using a variety of electron spectroscopies. This type of temperature dependence in the surface electronic structure is very similar to what is observed with our crystalline $\mathrm{P}(\mathrm{VDF}-\mathrm{TrFE})$ polymer films. In both cases, the electronic structure changes associated with the bulk are difficult to observe and, in both the case of $\mathrm{Ge}(100)$ [13] and $\mathrm{Si}(100)$ [14], and the present example of the ferroelectric polymer films, the increasing metallization with increasing temperature is restricted largely to the surface layer. This is, however, quite unlike other large crystaline molecular systems. The Cu-based dicyanoqinonediimine has a structure-dependent gap with the single crystals conducting down to $1.2 \mathrm{~K}$ [15] but the amorphous molecular films exhibit a semiconducting gap [16].

The ferroelectric transition in $\mathrm{P}(\mathrm{VDF}-\mathrm{TrFE})$ is associated with a rotation of the polar groups around the molecular chain axis and is connected with the disappearance of all-transchains to various combinations of cis (gauche) and transbonds with little or no net dipole moment [17]. This strongly suggests that, as in the case of $\mathrm{Ge}(100)$ [13] and $\mathrm{Si}(100)$ [14], electron-phonon coupling plays a strong role in the changes in electron structure with increasing temperature.

The most significant effect of temperature in the occupied electronic structure is the diminution of the photoemission feature at $12 \mathrm{eV}$ binding energy with increasing temperature, as seen in Fig. 2. We attribute this effect to dynamic scattering [18] of what may well be a molecular orbital associated with the C-F bond [19]. Following procedures used successfully elsewhere [18], the intensity as a function of temperature for this photoemission feature [Fig. 3(f)] can be employed to estimate an effective surface Debye temperature (not the true Debye temperature at the surface [18]) of 60 to $80 \mathrm{~K}$. This describes dynamic motions (phonon vibrations) normal to the surface. From inverse photoemission (Fig. 2), a similar diminution of the intensity of the lowest unoccupied molecular orbitals is also observed with increasing temperature, and a much lower effective Debye temperature of about $30 \mathrm{~K}$ can be estimated from the data below $300 \mathrm{~K}$. We can, therefore, conclude that either the effective surface Debye temperature is much lower than the bulk or that the Debye temperature below the surface ferroelectric phase transition is much lower than is the case when the surface is paraelectric and above the surface ferroelectric-paraelectric phase transition. Studies that probe the in-plane anharmonic dynamic motion in the surface layer are necessary to clarify this issue. Nonetheless, it is very clear from the very low effective Debye temperatures in the surface region that electron-phonon coupling does play a very important role in this transition. Theoretical calculations for molecular systems predict changes in metallicity due to dynamical effects [20,21].

In summary, we have undertaken the first combined photoemission and inverse photoemission study of the surface ferroelectric-paraelectric phase transition. It is very clear from this data that the surface ferroelectricparaelectric phase transition is accompanied by a change in electronic structure. This change in electronic structure is reflected by an increasing density of states at the Fermi energy and the onset of an increasing conductivity with 
increasing temperature. The surface ferroelectric phase is quite distinct from the bulk. Since the composition does not change from the surface to the bulk in a molecular crystal, we suggest that the differences between the surface and bulk electronic structure must be related to the lower coordination of the surface, which, in turn, leads to a different surface free energy and greater dynamic motion at the surface.

This work was supported by the National Science Foundation (DMR-92-21655, DMR-94-96131, and ECS9616246) and the Nebraska Research Initiative through the Center for Materials Research and Analysis, S.P.P. and N.P. were supported by INTAS Project No. N93-1700.

*Corresponding author: Department of Physics and Astronomy and the Center for Material Research and Analysis, Behlen Laboratory of Physics, University of Nebraska, Lincoln, Nebraska 68588-0111.

Electronic address: pdowben@unlinfo.unl.edu

[1] D. N. McIlroy, J. Zhang, S.-H. Liou, and P. A. Dowben, Phys. Lett. A 207, 367 (1995).

[2] J.-H. Park et al., J. Appl. Phys. 79, 4558 (1996); Phys. Rev. Lett. 76, 4215 (1996).

[3] D. H. McIlroy, C. Waldfried, J. Zhang, J.-W. Choi, F. Foong, S.-H. Liou, and P. A. Dowben, Phys. Rev. B 54, 17438 (1996).

[4] J. W. Choi, S.-H. Liou, P. A. Dowben, J. Zhang, and E. W. Plummer (to be published).

[5] A. V. Bune, V. M. Fridkin, S. Ducharme, L. M. Blinov, S.P. Palto, A. Sorokin, S. G. Yudin, and A. Zlatkin, Nature (London) (to be published).

[6] P. A. Dowben, D. N. McIlroy, and D. Li, Surface Magnetism of the Lanthanides, Handbook on the Physics and Chemistry of the Rare Earths (Elsevier, Amsterdam, 1997), Vol. 24, Chap. 159, and references therein.

[7] S. H. Liu and R.A. Klemm, Phys. Rev. Lett. 73, 1019 (1994).

[8] S. Palto, L. Blinov, A. Bune, E. Dubovik, V. M. Fridkin, N. Petukhova, K. Verhhovskaya, and S. Yudin, Ferroelectr. Lett. 19, 65 (1995); L. M. Blinov, Sov. Phys. Usp. 31, 623 (1988).

[9] P. W. Erdman and E. C. Zipf, Rev. Sci. Instrum. 53, 225 (1982).

[10] A. Bune et al., Appl. Phys. Lett. 67, 3975 (1995); L. M. Blinov et al., Thin Solid Films 284-285, 474 (1996); S. Ducharme, A. Bune, L. M. Blinov, V. M. Fridkin, S. P. Palto, A. V. Sorokin, and S. G. Yudin, Phys. Rev. B 57, 25 (1998).

[11] D. N. McIlroy, C. Waldfried, T. McAvoy, J. Choi, P. A. Dowben, D. Heskett, Chem. Phys. Lett. 22, 1048 (1997).

[12] B. Dardel et al., Europhys. Lett. 19, 525 (1992).

[13] S. D. Kevan and N. G. Stoffel, Phys. Rev. Lett. 53, 702 (1984); S. D. Kevan, Phys. Rev. B 32, 2344 (1985).

[14] L. Gravioli, M. G. Betti, and C. Mariani, Phys. Rev. Lett. 77, 3869 (1996).

[15] A. Aumüller et al., Ang. Chem. 98, 759 (1986) [Angew. Chem. Int. Ed. Engl. 25, 740 (1986)].

[16] D. Schmeißer et al., Chem. Phys. Lett. 148, 423 (1988).

[17] T. Furukawa, Phase Transit. 18, 143 (1989).

[18] C. Waldfried, D. N. Mcllroy, J. Zhang, P. A. Dowben, G. A. Katrich, and E. W. Plummer, Surf. Sci. 363, 296 (1996).

[19] P. A. Dowben and M. Grunze, Ber. Bunsenges. Phys. Chem. 85, 728 (1981).

[20] G. Santoro, L. Guidoni, A. Parola, and E. Tosatti, Phys. Rev. B 55, 16168 (1997).

[21] X. Xia, X. Li, D. L. Lin, and T. F. George, Phys. Rev. B 42, 4790 (1990). 